\title{
Five personality dimensions in patients with irritable bowel syndrome
}

\author{
Alireza Farnam \\ Mohammad H Somi \\ Firouz Sarami \\ Sara Farhang \\ Liver and Gastrointestinal Diseases \\ Research Center, Tabriz University \\ of Medical Sciences, Tabriz, Iran
}

\begin{abstract}
Aim: Irritable bowel syndrome (IBS) as a functional disorder of the gastrointestinal tract has been related to psychological factors. Aim of this study is to study the differences of personality factors between IBS patients compared to our general population.

Methods: This study was performed in clinics of Tabriz Medical University during 2006-2007. IBS was diagnosed using the Rome II diagnostic criteria after exclusion of organic bowel pathology. The entry of each patient was confirmed following a psychiatric interview and after any comorbid psychiatry disorder was ruled out. Personality traits and score of each factor was evaluated using NEO five factor personality inventory compared to results of a previous study on general population of Iran.

Results: One hundred and sixty six patients were studied. The mean age $( \pm \mathrm{SD})$ of them was $33.6( \pm 11.4)$ years $(60.8 \%$ female $)$. Our study population had their symptoms for a mean interval of 47.3 month. The bowel problems were provoked by distress in more than $80 \%$ of patients.

Pain in female patients was reported to be more frequent but both gender described the association between stressors and their symptoms. NEO Five-Factor Inventory showed a significantly higher level of neuroticism and conscientiousness and lower level of openness and agreeableness in theses nonpsychiatric IBS patients. Women with IBS had significantly higher levels of openness, conscientiousness, and extraversion compared to men.
\end{abstract}

Conclusion: Differences were observed between IBS patients and general population. Patients with IBS may benefit from psychological interventions.

Keywords: irritable bowel syndrome, personality, five-factor model

\section{Introduction}

The common functional gastrointestinal disorder, irritable bowel syndrome (IBS), has a wide variety of presentations (Camilleri and Choi 1997). The etiology is unclear and the syndrome is classified as "functional" due to absence of any organic causes for symptoms. There is a growing body of evidence that it is influenced by psychosocial stressors. The high rates of psychiatric comorbidity in IBS patients indicate that affective symptoms may be a specific and integral part of the syndrome, rather than a specific syndrome related to a chronic intestinal disease (Walker et al 1992).

The incidents of mood and anxiety disorders have been practically well studied in the IBS patient using up-to-date procedures and has resulted in recommendations on how to best detect and integrate their treatment to achieve a better outcome (Drossman et al 1999) and have led to significant improvements in treatment. Psychotherapeutic intervention for patients with comorbid psychiatric disorders is valuable. Underlying personality structure was mislaid in recognizing IBS may be due to the poor quality and doubtful results of early researches (Olden 2003). Personality, as an almost constant characteristic, is a matter of interest influencing pain duration, severity, and functional impairment in different diseases but very little is known about personality in IBS. 
The NEO five-factor personality inventory is a widely accepted model because the structure of traits in it is consistent among highly diverse cultures with distinct languages and between men and women, and older and younger adults (Costa and McCrae 1997). This model of personality defines personality traits in terms of five basic dimensions. These dimensions are: extraversion (talkativeness, assertiveness, activity; vs silence, passivity and reserve); agreeableness (kindness, trust, and warmth vs selfishness and distrust); conscientiousness (organization, thoroughness, and reliability vs carelessness, negligence and unreliability); neuroticism (nervousness, moodiness, temperamentality vs emotional stability); and openness to experience (imagination, curiosity, and creativity vs shallowness and imperceptiveness).

There is a serious lack of information on the psychiatric characteristics of IBS patients in the Iranian population. We wished to assess the distribution of personality traits in IBS patients of our community and compare them to Iranian general population and hypothesize significant differences between them.

\section{Methods}

Continuous patients attended to university out-patient clinics with a diagnosis of IBS were included. Patients of the current report are part of a large study evaluating nonpsychiatric IBS patients. The diagnosis was established after a stool exam, clinical evaluation, and endoscopy (in some cases) by a gastroenterologist using ROME II criteria for IBS. All patients were clinically investigated to identify the presence of "alarm factors." Patients were excluded if an organic cause of the condition was possible, or if there was a history of serious somatic disease. All patients gave a written consent. Demographic information, severity and course of illness, and abdominal pain severity over the past week were obtained.

The patients were referred to the first author for psychiatric and psychosomatic assessments, blinded to characteristics of IBS. A history or current symptom of any DSM-IV psychiatric diagnosis on Axis I or seizure disorders also led to exclusion from the study.

Personality dimensions in nonpsychiatric IBS patients were evaluated by the NEO Five-Factor Inventory (NEO-FFI), a 60-item questionnaire which usually requires 10 to 15 minutes to complete. This questionnaire is rated on a five-point scale to yield scores in five major domains of personality and requires a sixth-grade reading level. Scores of five personality factors measured by NEO are previously described in the Iranian population (Garousi et al 2001). This large scale study determined personality factors in
2000 randomly selected individuals (without established psychiatric disorders) from different provinces of Iran by NEO-FFI.

Data analysis was performed using SPSS $^{\circledR}$ version 13 statistical package (SPSS Inc., Chicago, IL). The $\chi^{2}$ test and student's $t$ were used to determine the significance of associations between different variables. One sample $t$ test was used to compare our sample with Iranian general population. The overall significance was set at 0.05 .

\section{Results}

One hundred and sixty six patients with irritable bowel syndrome were enrolled in this study. The mean age $( \pm \mathrm{SD})$ of them was $33.6( \pm 11.4)$ years $(60.8 \%$ female). Our study population had their symptoms for a mean interval of 47.3 month. The bowel problems were provoked by distress in more than $80 \%$ of patients. Such symptoms interrupt their daily activities like patients' job (18.1\%), education (7.2\%), family $(25.3 \%)$, and social communications $(5.4 \%)$ or a combination of these (13.9\%).

Forty-six percent of patients experienced abdominal pain less than 3 times a day, $29.4 \% 3$ to 6 times, and $24.5 \%$ more than 6 times a day. Pain in female patients was reported to be more frequent $(p=0.004)$, but both gender described the positive association between emotional stressors and their symptoms $(p=0.179)$. Mean age or mean duration of the symptoms were not significantly different between male and females.

NEO-FFI showed a significantly higher level of neuroticism and conscientiousness and lower level of openness and agreeableness in nonpsychiatric IBS patients. Table 1 shows the mean scores of five personality factors in our patients compared to Iranian general population (by NEO FFI) (Garousi et al 2001). Women with IBS had significantly higher levels of openness (27.18 vs 25.67 in male, $p=0.042$ ), conscientiousness ( 32.93 vs 31.57 in male, $p=0.022$ ), and

Table I Mean (SD) scores of five personality factors measured by NEO five-factor inventory in patients with irritable bowel syndrome (IBS) compared to Iranian general population

\begin{tabular}{llll}
\hline & IBS patients & $\begin{array}{l}\text { General } \\
\text { population }\end{array}$ & $\mathbf{p}$ \\
\hline Openness & $26.95(5.32)$ & $27.94(4.87)$ & $<\mathbf{0 . 0 0 0 5}$ \\
Conscientiousness & $32.87(7.74)$ & $31.62(5.64)$ & $\mathbf{0 . 0 3 9}$ \\
Extraversion & $26.88(6.16)$ & $26.89(6.15)$ & 0.983 \\
Agreeableness & $29.33(6.78)$ & $32.90(7.00)$ & $<\mathbf{0 . 0 0 0 5}$ \\
Neuroticism & $26.27(9.44)$ & $22.92(9.54)$ & $<\mathbf{0 . 0 0 0 5}$ \\
\hline
\end{tabular}




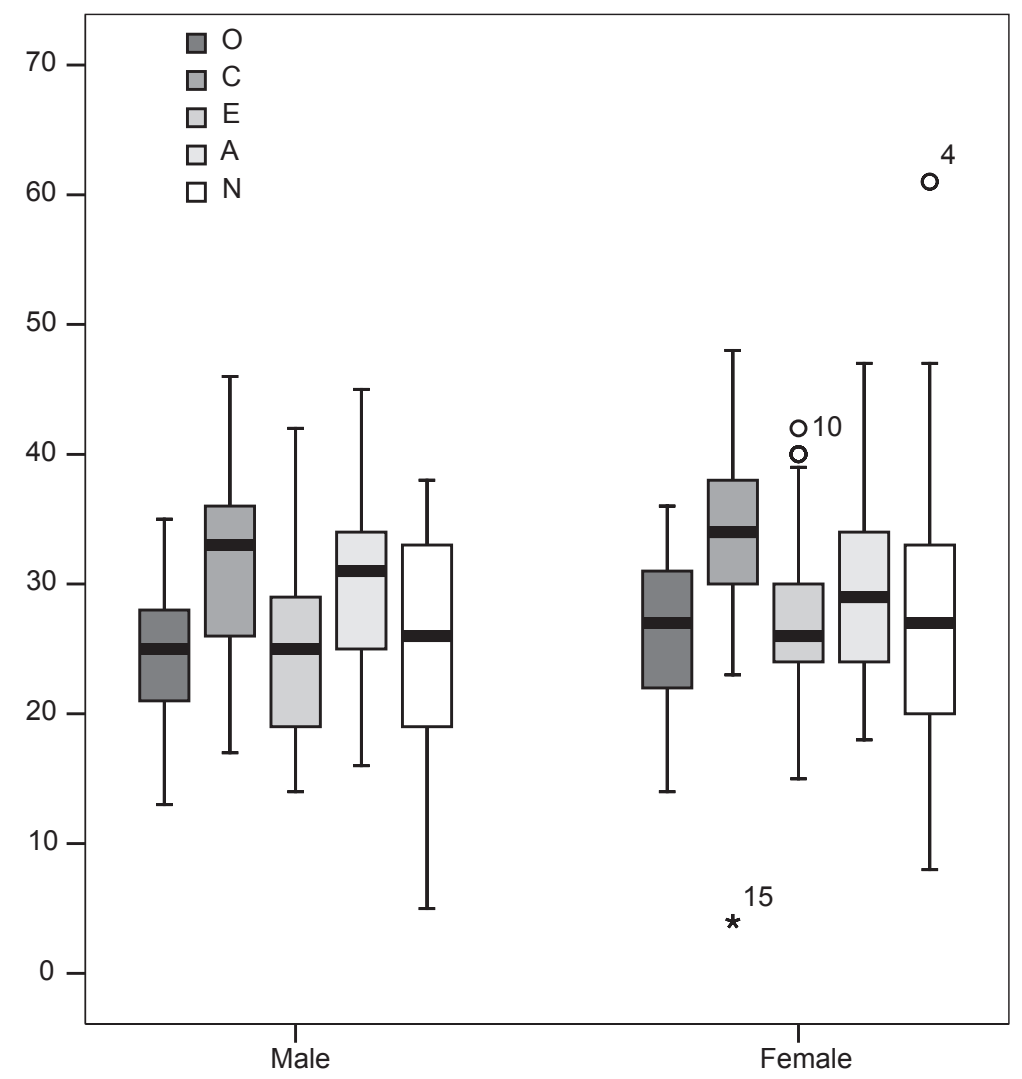

Figure I Scores of personality factors in irritable bowel syndrome patients defined by gender.

Abbreviations: $\mathrm{O}$, openness to experience; $\mathrm{C}$, conscientiousness; $\mathrm{E}$, extraversion; $\mathrm{A}$, agreeableness; $\mathrm{N}$, Neuroticism.

extraversion ( 26.51 vs 25.65 in male, $\mathrm{p}=0.014)$ compared to men (shown in Figure 1).

\section{Discussion}

The present manuscript, as a part of a larger study was designed to investigate the personality characteristics of nonpsychiatric IBS patients. Emotional states and personality traits may affect the physiology of the gut (Guthrie et al 1991), play a part in how symptoms are experienced and interpreted and can influence treatment (Bennet et al 1998; Wooda 1999). This can be obliging while considering the management strategy to achieve a better outcome in IBS patients which the prevalence has been reported to be $18.4 \%$ in our population (Drossman et al 1999).

The five-factor model provides a dimensional account of the structure of normal personality traits, dividing personality into the five broad dimensions. Neuroticism and aggression are reported to be higher in patients with functional gastrointestinal disease without psychiatric comorbidity and personality traits are believed to influence pain reports (Tanum and Malt 2001). A low level of neuroticism and little concealed aggressiveness is reported to predict treatment outcome with the antidepressants in nonpsychiatrics, which was most prominent in women. These personality dimensions were better predictors of outcome compared to serotonergic sensitivity (Fock et al 2001).

Differences between male and female patients with IBS were noticed elsewhere. Significant differences found in the trait neuroticism, extraversion, and conscientiousness was consistent with other studies that suggest women consistently score higher than men on self-reported trait anxiety (Talley et al 1990; Tran et al 2006).

The data for nonpsychiatric individuals drawn from a pool prepared for standardization of the Iranian version of NEO PI-R are among limitations of the present study.

In the current study IBS patients scored higher in neuroticism and conscientiousness and scored lower in agreeableness and openness. Neuroticism is a personality trait characterized by overstated reactivity to physiological changes and unstable emotions. It is also related to emotional regulation, motivation, and interpersonal skills. Centrally targeted medications, such as anxiolytics and low-dose tricyclic antidepressants, which involve inhibitory effects on the sensitivity of the emotional motor system 
(Drossman et al 2002), are widely used in IBS patients and can balance the neuroticism dimension of such patients.

IBS patients with high self-consciousness may benefit from selective serotonin re-uptake inhibitors which increase the extracellular level of the neurotransmitter serotonin. A systematic review on the effectiveness of psychological treatments in IBS is currently being undertaken (Creed and Guthrie 1989), a procedure which may help balance the agreeableness and openness dimensions of personality.

The present study gives added evidence of differences between IBS patients and the general population. This may help to focus the management plan to obtain a better outcome. There are evidences of differences of personality dimensions according to symptom-based subtypes of IBS (Tanum and Malt 2000), which will be considered in our later studies.

\section{Disclosure}

The authors report no conflicts of interest in this work.

\section{References}

Bennett EJ, Palmer K, Badcock C-A, et al. 1998. Functional gastrointestinal disorders: psychological, social, and somatic features. Gut, 42:414-20.

Camilleri M, Choi MG. 1997. Review article: irritable bowel syndrome. Aliment Pharmacol Ther, 11:3-15.

Creed F, Guthrie E. 1989. Psychological treatment of the irritable bowel syndrome: a review. Gut, 30:1601-9.

Costa PT, McCrae RR. 1997. Stability and change in personality assessment: The revised NEO Personality Inventory in the year 2000. J Pers Assess, 68:86-94.
Drossman DA, Camilleri M, Mayer E, et al. 2002. AGA technical review on irritable bowel syndrome. Gastroenterology, 123:2108-31.

Drossman DA, Corazziari E, Talley NJ, et al. 1999. Rome II: a multinational consensus document on functional gastrointestinal disorders. Gut, 45(Suppl. II):II1-81.

Drossman DA, Creed FH, Olden KW, et al. 1999. Psychosocial aspects of the functional gastrointestinal disorders. Gut, 45(Suppl. II): II25-II30.

Olden KW. 2003. Irritable bowel syndrome: What is the role of the psyche? Dig Liver Dis, 38:200-1.

Fock KM, Chew CN, Tay LK, et al. 2001. Psychiatric Illness, Personality Traits and the Irritable Bowel Syndrome. Ann Acad Med Singapore, 30:611-14.

Garousi MT, Mehryar AH, Tabatabi MG. 2001. Application of the NEO PIR test and analytic evaluation of its characteristics and factorial structure among Iranian university students. Human Sci Alzahra Uni, 11:30-8.

Guthrie E, Creed F, Dawson D, et al. 1991. A controlled trial of psychological treatment for the irritable bowel syndrome. Gastroenterology, 100:450-7.

Talley NJ, Phillips SF, Bruce B, et al. 1990. Relation among personality and symptoms in nonulcer dyspepsia and the irritable bowel syndrome. Gastroenterology, 99:327-33.

Tanum L, Malt UF. 2000. Personality traits predict treatment outcome with an antidepressant in patients with functional gastrointestinal disorder. Scand J Gastroenterol, 35:935-41.

Tanum L, Malt UF. 2001. Personality and physical symptoms in nonpsychiatric patients with functional gastrointestinal disorder. J Psychosom Res, 50:139-46.

Tran Y, Craig A, Boord P, et al. 2006. Personality traits and its association with resting regional brain activity. Int $J$ Psychophysiol, 60:215-24.

Walker EA, Katon WJ, Jemelka RP, et al. 1992. Comorbidity of gastrointestinal complaints, depression, and anxiety in the Epidemiologic Catchment Area (ECA) Study. Am J Med, 92(1A):26S-30S.

Wooda JD, Alpersb DH, Andrews PL. 1999. Fundamentals of neurogastroenterology. Gut, 45(Suppl 2):II6-II16. 